European Journal of Obstetrics \& Gynecology and

Reproductive Biology 79 (1998) 199-204

\title{
Activity of smooth muscles in human cervix and uterus
}

\author{
Marjan Pajntar.*, Ivan Verdenik, Stanko Pušenjak, Drago Rudel, Brane Leskošek \\ 'Department of Obstetrics and Gynaecology. University Medical Centre Šlajmerjeva 3. Ljubljana. Slovenia "Institute \\ for Social Medicine. Faculty of Medicine. University of Ljubljana. Zaloška 4. Ljubljana. Slovenia \\ Received 7 August 1997; received in revised form 17 November 1997; accepted 12 February 1998
}

Abstract

Objective: To find the similarities and dissimilarities between the activity of the human smooth muscles in the cervix and in the uterine corpus at the onset of induced labour. Study design: Electromyographic activity was measured in 14 primiparous women after amniotomy. The data were sampled and stored digitally in real time. For statistical analysis. the first $20 \mathrm{~min}$ of recordings following amniotomy were analyzed. The ratio between the mean activity at agiven time and the mean activity over the whole 20 min was used for the comparison between the cervical and uterine activity. Results: The analysis of correlation showed that the electromyographic activity in the cervix differed from that in the uterine corpus in the majority of the enrolled cases. Cone/usions: The muscular activity in the cervix is independent of that in the uterine corpus at the onset of induced labour. ( 1998 Elsevier Science Ireland Ltd.

Keywords: Electromyography; Cervix; Uterus; Smooth muscles; Labour

\section{Introduction}

The activity and the role of the cervix during pregnancy and labour has not been thoroughly investigated yet. We believe that the cervix in pregnancy has to remain closed and retain the conceptus, while during labour it should dilate and retract. Thus, during pregnancy the smooth muscles in the cervix (spiral or circular fibres) should by contracting contribute to the closure of the cervical canal, whereas during labour other smooth muscles (longitudinal fibres) should actively contribute to the dilation and retraction of the cervix, although through a different kind of contraction [1]. To perform this function, the smooth cervical muscles lying more or less in a circular pattern should act independently of those in the uterine corpus. This independent activity has already been observed in animals. The cervical and uterine smooth muscles appear to act independently in ewes, possibly reflecting their separate functions [2]. There exist some clinical evidence

\footnotetext{
*Corresponding author. Tel./fax: + 38661 315328; e-mail: marjan. pajntar@kclj.si
}

that the cervix in humans constricts, constricts rhythmically and constricts independently of the corpus [3-6]. This activity can be assessed reliably by electromyography.

Over the last few decades, uterine electromyographic (EMG) activity has been investigated in animals and in humans. Recordings made either at the myometrial or abdominal level provide reliable and useful information on uterine muscle behaviour [7,8]. Most studies measured the EMO activity in the uterine corpus, whereas our group have focused on measuring the cervical EMO activity [9-11].

Our previous study indicated the possibility of a difference between EMO activity in the human cervix and that in the uterine corpus [9]. The aim of the present study was to obtain evidence of differences between the EMO activity in the cervix and in the uterine corpus in humans.

\section{Materials and methods}

The investigation was approved by the 'National Medical Ethics Committee', and informed consent was obtained from all patients. 


\subsection{Study population}

The study involved 14 primiparous women at term visiting the delivery room for induced labour who consented to participate in the study. The CTG showed no or infrequent uterine contractions. After admission to the delivery room, cervical ripeness was estimated according to the Bishop score (me an 6.46, S.D. 1.02), and an amniotomy was performed. The mean ini ti al cervical dilatation was $25.0 \mathrm{~mm}$ (S.D.=0.63). An open-end catheter (Hewlett-Packard) for measuring and recording the intrauterine pressure was inserted in the uterine cavity, and an ECG electrode was attached to the infant's head to monitor the fetal heart rate (CTG monitoring).

\subsection{EMG recordings}

EMG activity of the uterine corpus was detected using three surface skin Ag-AgCI di se electrodes (In Vivo Metrics Inc.) 5 $\mathrm{mm}$ in diameter. The skin was cleaned and treated with electrode gel. The electrodes were attached in su eh a way as to allow measurements of EMG activity in two directions, transversely and longitudinally, perpendicular to each other, with the reference electrode placed in the middle (Fig. 1, up per part). The interelectrode distance was $7 \mathrm{~cm}$. The lower electrodes were placed $-5 \mathrm{~cm}$ above

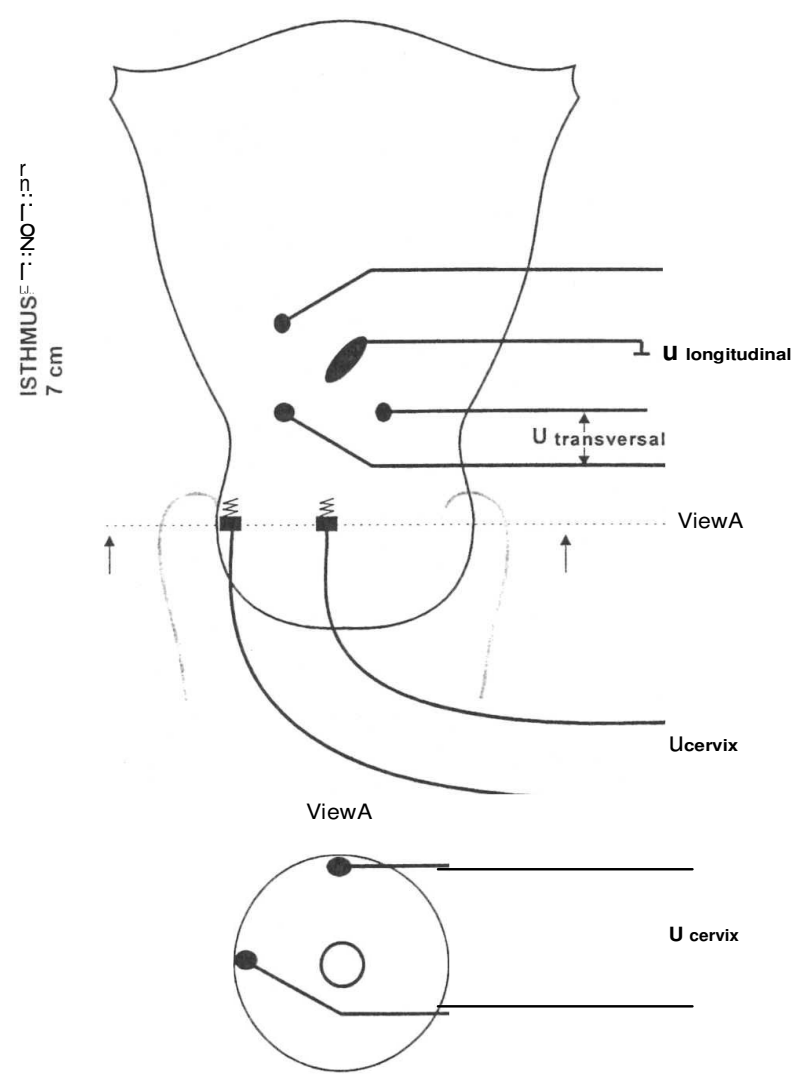

Fig. 1. Electrode positioning on the uterine corpus and in the cervix. the symphysis. To record cervical EMG activity, a pair of spiral needle electrodes (Hewlett-Packard) was used. The electrodes were attached to the cervix in the proximal part of the portio (at 09:00 hand 12:00 h) for detection in the transverse (circular) direction (Fig. 1, lower part). The calculated initial interelectrode distance was $31.7 \mathrm{~mm}$ (S.D.=0.63). At the end of the observed interval it was 33.9 $\mathrm{mm}$ (S.D.=0.70). A reference electrode was placed on the woman's thigh.

\subsection{Amplification, filtering and sampling}

Three differential preamplifiers $(A=1000, C M R R>90 \mathrm{~dB}$, bandwidth=O.1 Hz-1000 Hz) were used, two for uterine EMG and one for cervical EMG. The signals from preamplifiers were fed to an amplifier with a built-in low-pass filter (cutoff frequency $4.5 \mathrm{~Hz}, 60 \mathrm{~dB} /$ decade) with an additional userselected gain $(A=0.5$ to 10$)$. A paper recorder was used to monitor EMG activity. Amplified and low-pass filtered EMG signals were digitised in real time by a PC-based 8-channel, 12bit $A I D$ converter. The sampling frequency was $20 \mathrm{~Hz}$. The data were written on a hard disk concurrently with the sampling.

\subsection{Processing and analysis}

For each labour, EMG signals, recorded during the first 20 min after amniotomy, were processed. First, digital filtering $(0.1 \mathrm{~Hz}-4 \mathrm{~Hz})$ was applied. Second, the root me an square (RMS) values were calculated for each 5-s interval, according to the equation given by Ramondt et al. [13]. An example of the procedure implementation is shown in Fig. 2. The upper trace represents raw EMG signal, the second trace the EMG signal after filtering, and RMS values in the third trace. It can be seen that the RMS corresponds closely to the envelope of the raw EMG.

Since the comparison of absolute values would be almost meaningless, we decided to compute (for each minute) relative activity, where, the relative, means relative to the average EMG during the recording interval $(20 \mathrm{~min})$. Instances with EMG amplitudes higher than half of the average are shown on the fourth trace of Fig. 2. Statistical correlation of these 20 values from one source and 20 from the other thus represents the measure of temporal relationship between the signals.

\subsection{Statistical analysis}

Pearson correlation coefficients were derived from minute values for all three signal pairs, namely, cervix vs. uterus longitudinally, cervix vs. uterus transversely, and uterus longitudinally vs. uterus transversely in each of 14 cases. To compute the average correlation coefficient for a given pair, Pearson's $r$ values were transformed into Fisher $z$ values. The mean correlation coefficients for $20 \mathrm{~min}$ of measurement for the three pairs were calculated as the 

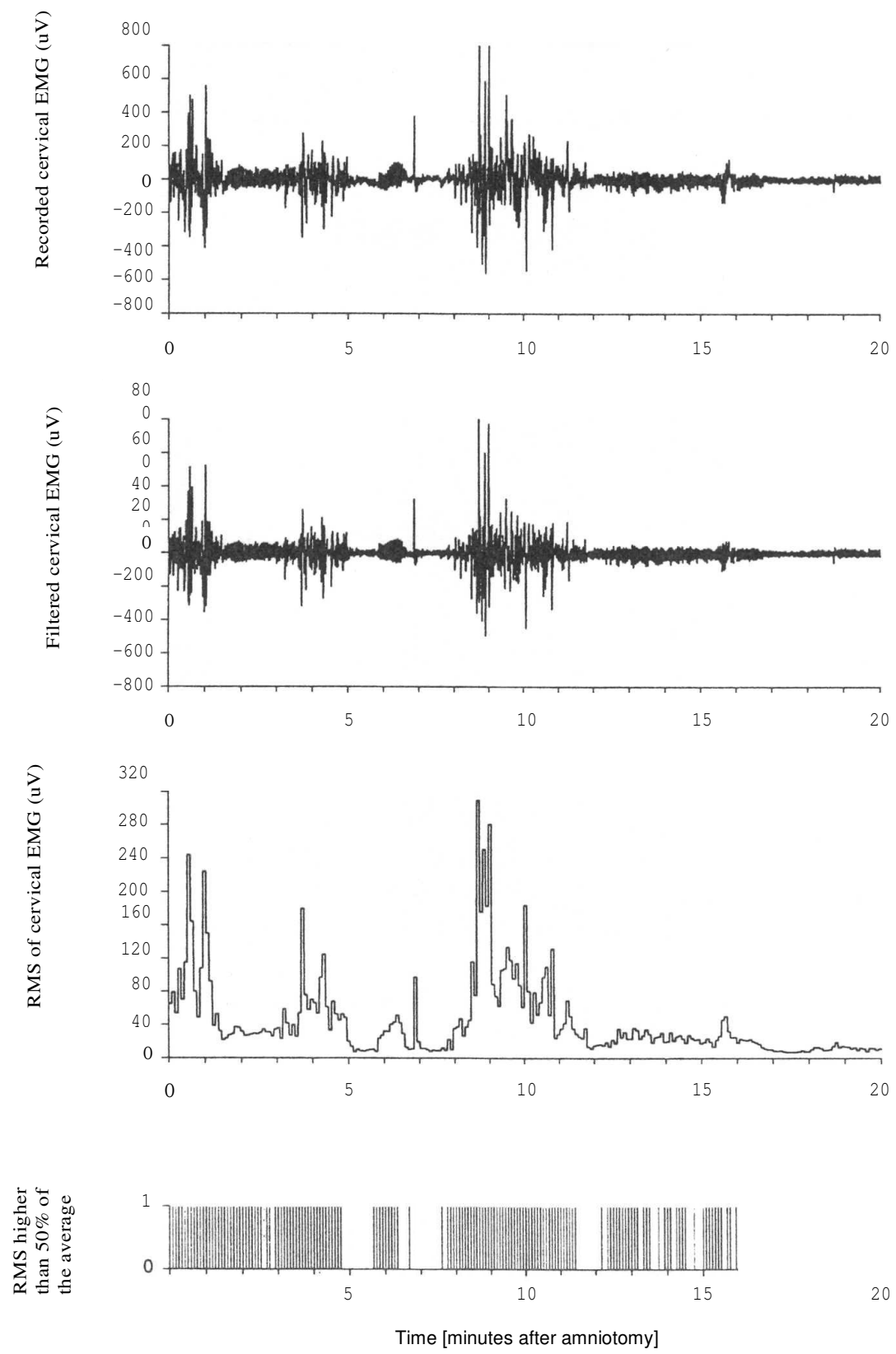

Fig. 2. Illustration of data processing.

weighted arithmetic me an to obtain the final correlation between the pairs.

Software used for signal processing was MATLAB and for statistics SPSS.

\section{Results}

Fig. 3 shows three signals recorded during the first $20 \mathrm{~min}$ after amniotomy on one of the patients plus corresponding trace of intrauterine pressure. It can be seen that activity of the cervix is by and large different than those in both uterine directions. We could observe some similarity between electric al uterine and mechanical activity although in the beginning of labour not particularly pronounced.

In 11 of the 14 cases no significant correlations were found between the electrical activity (RMS) of the cervix and of the uterine corpus measured transversely (Table 1). The mean correlation coefficient for the whole group was small $(\mathrm{r}=0.161)$ and not significant.

Similarly, in 10 of the 14 cases no significant correlations were found between the electrical activity (RMS) of 
M. Pajntar et al. I European Journal of Obstetrics \& Gynecology and Reproductive Biology 79 (1998) 199-204

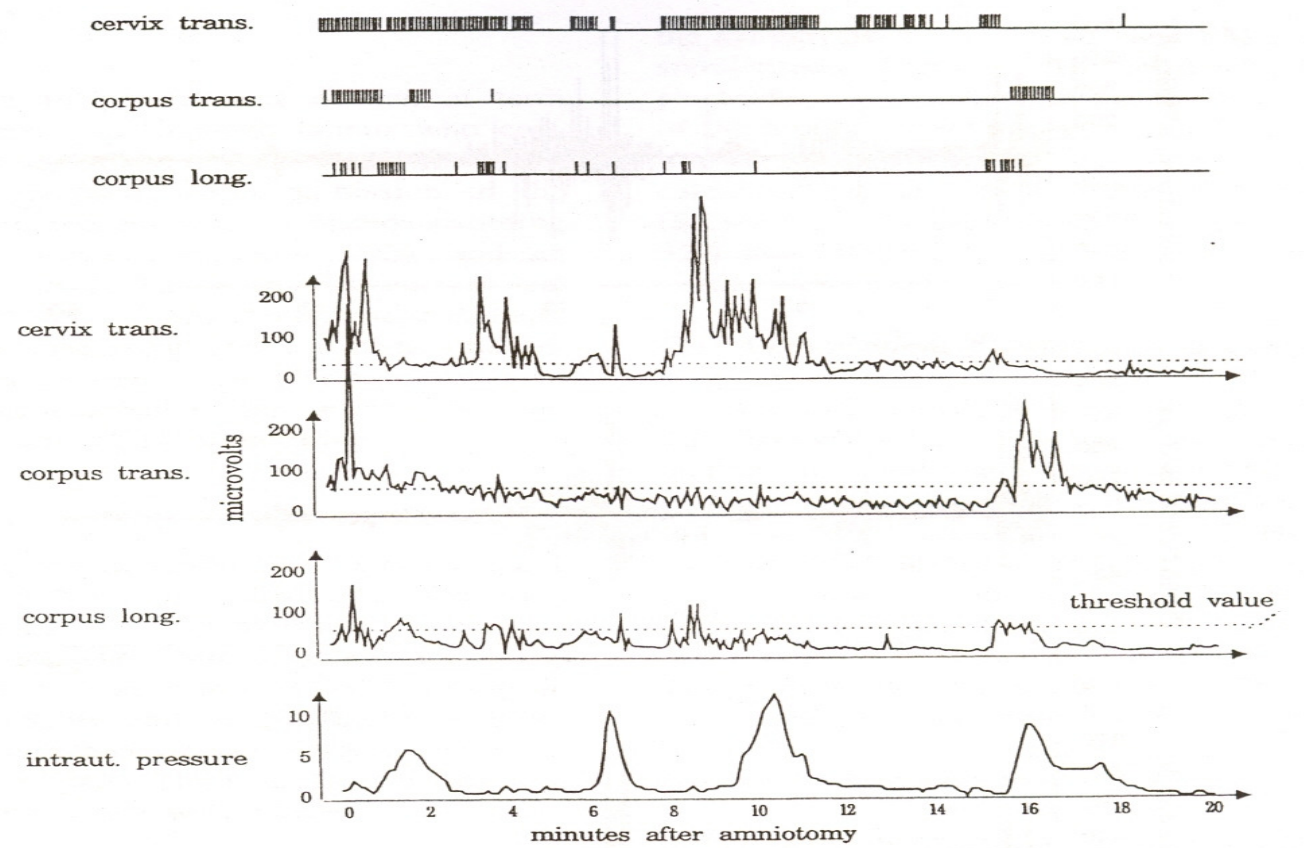

Fig. 3. RMS of the EMG and corresponding relative amplitudes in 20-min interval after amniotomy - patient BM.

the cervix and of the uterine corpus measured longitudinally (Table 2). The mean correlation coefficient for the whole group was small $(r=0.140)$ and not significant.

Conversely, in 11 of the 14 cases significant correlations were found between the electrical activity (RMS) in two directions in the uterine corpus (Table 3 ). The mean correlation coefficient for the whole group was high (0.692) and statistically significant $(P<0.001)$.

Table I

Correlation between cervical and uterine corpus (transverse) RMS of the EMG

\begin{tabular}{|c|c|c|c|c|c|c|c|}
\hline$\underline{\text { Patient }}$ & Pearson's $I^{\prime}$ & $P$ & Mean correlation & Patient & Pearson's $r$ & $P$ & Mean correlation \\
\hline $\mathrm{BM}$ & 0.19 & 0.40 & $1^{\prime}=0.161$ & $\mathrm{BM}$ & 0.42 & 0.06 & $\mathrm{r}=0.140$ \\
\hline $\mathrm{BN}$ & -0.14 & 0.54 & $P=N . S$ & DA & -0.01 & 0.98 & $P=N . S$ \\
\hline DA & 0.01 & 0.98 & & OB & 0.01 & 0.98 & \\
\hline OB & 0.37 & 0.15 & & OM & -0.02 & 0.92 & \\
\hline $\mathrm{OM}$ & 0.09 & 0.69 & & PB & 0.11 & 0.64 & \\
\hline PB & -0.08 & 0.71 & & PV & -0.03 & 0.90 & \\
\hline PV & -0.41 & 0.07 & & $\mathrm{RJ}$ & -0.22 & 0.34 & \\
\hline SD & -0.03 & 0.89 & & TM & -0.06 & 0.79 & \\
\hline $\mathrm{TM}$ & -0.07 & 0.74 & & $\mathrm{TN}$ & 0.36 & 0.11 & \\
\hline $\mathrm{TN}$ & 0.17 & 0.46 & & $\mathrm{KH}$ & 0.53 & 0.015 & \\
\hline $\mathrm{KH}$ & 0.51 & 0.022 & & $\mathrm{BN}$ & -0.56 & 0.009 & \\
\hline RV & 0.62 & 0.003 & & RV & 0.58 & 0.007 & \\
\hline SM & 0.84 & 0.001 & & SM & 0.91 & 0.001 & \\
\hline
\end{tabular}

\section{Discussion}

Our aim was to detect similarities and dissimilarities in the activities of the smooth muscles in the cervix and uterine corpus at the onset of induced labour. Electromyography measures the changes occurring in the membrane electrical potential of the myometrium. Other studies have confirmed the close relationship between

Table 2 Correlation between cervical and uterine corpus (longitudinal) RMS of the

N.S., not significant. 
Table 3

Correlation between utenne corpus RMS of the EMG measured in two directions

\begin{tabular}{|c|c|c|c|}
\hline Patient & Pearson's $r$ & $\underline{P}$ & Mean correlation \\
\hline $\mathrm{KH}$ & 0.88 & 0.001 & \multirow{14}{*}{$\begin{array}{l}0.692 \\
P<0.001\end{array}$} \\
\hline $\mathrm{OM}$ & 0.65 & 0.002 & \\
\hline $\mathrm{PB}$ & 0.55 & 0.011 & \\
\hline PV & 0.64 & 0.002 & \\
\hline $\mathrm{R} 1$ & 0.49 & 0.027 & \\
\hline so & 0.92 & 0.001 & \\
\hline SM & 0.95 & 0.001 & \\
\hline $\mathrm{TM}$ & 0.99 & 0.001 & \\
\hline DA & 0.89 & 0.001 & \\
\hline $\mathrm{TN}$ & 0.75 & 0.001 & \\
\hline RV & 0.75 & 0.001 & \\
\hline $\mathrm{BN}$ & 0.34 & 0.143 & \\
\hline $\mathrm{BM}$ & 0.42 & 0.069 & \\
\hline $\mathrm{OB}$ & 0.47 & 0.058 & \\
\hline
\end{tabular}

electrical and mechanical activity In the uterine smooth muscles $[8,12]$. According to Devedeux et al. [8J, smooth muscle EMG could be divided into slow wave (below $0.03 \mathrm{~Hz}$ ) and fast wave $(0.1-3 \mathrm{~Hz})$ bands. In the slow wave band, it is al most impossible to differentiate between uterine activity and mechanical artefacts such as skin stretching. To avoid artefacts, this study focused on the fast wave frequency band alone.

Some authors reporting on the uterine EMG activity rely on 'EMG bursts' in analysing signals in the time domain [12,13J. Bursts are usually defined as series of spikes of a certain amplitude and frequency. However, we have found that at the on set of the induced labour, the EMG activity is irregular and is not grouped into pronounced bursts (also the contractions are weak and irregular). Therefore, the quantitative measures such as burst duration and intensity of bursts, are not adequate. Thus the method was considered inappropriate for our study. The RMS definition of EMG activity at any gi ven time, because of its mathematical nature, seemed more appropriate.

As the type $\mathrm{s}$ of electrodes and their arrangement and attachment in the uterine corpus and cervix were different. it was clear that the comparison of RMS amplitudes could not lead to reliable conclusions. Therefore, relative values were compared instead.

In the observed period the assessed cervical interelectrode distance increased for less than $10 \%$ on average. Presuming that the amount of muscle cells between the electrodes remained almost equal, the conditions for EMG recording during the observed period were considered constant.

The difference between the EMG activity in the cervix and uterine corpus was studied by analysing 1-min records. The analysis showed that in most parturient women under observation, the EMG activity in the cervix was different from that in the uterine corpus, regardless of whether the latter was measured transversely or longitudinally.

The insignificant correlation coefficient between two sources of EMG signals (Table 1 and Table 2)proved that no common source of variability in EMG signal s existed. The significant correlation coefficient (Table 3) indicates the same source of variability.

The analysis of differences in the EMG activity in the uterine corpus detected in two different directions served primarily as a control parameter. It was found that in most women the RMS of the EMG activities correlated significantly, and that a major part of the variance originated from the same source.

The present study supports the findings of some clinical observations [3-6] and previous electromyographic studies on a few cases only [8] that in humans the smooth muscles in the cervix act independently of those in the uterine corpus. We studied the situation at the onset of induced labour (after amniotomy), in relatively unripe cervices, i.e. when, in our opinion, cervical smooth muscles, whose fibres lie either in spiral or circular directions, are still active, as they help to keep the cervical canal closed during pregnancy. This independent activity of the cervical smooth muscles is important for better understanding of the role of the cervix in normal pregnancy and labour, in preterm delivery and in prolonged pregnancy. From the clinical point of view to know the intensity of the activity of the smooth muscle fibres in the cervix, is of extreme importance for predicting the course of the latent and active phase of labour, and consequently for appropriate management of labour.

\section{Condensation}

Smooth muscles of the relatively unripe cervices act independently of those in the uterine corpus at the onset of induced labour.

\section{References}

[1] Pajntar M. The smooth muscles of the cervix in labour. Eur JObstet Gynecol Reprod Biol 1994:55:9-12.

[2] Stys S. Clewell WHo Mechia G. Changes in cervical compliance at partuntion independent of uterine activity. Am JObstet Gynecol 1978;130:414-8.

[3] Nixon WXW. Uterine action. normal and abnormal. Am JObstet Gynecol 1951 ;62:964-8 .

[4] Theobald. GW. Endocnne control of utenne innervation. London: Bunerworth. 1973.

[5] Danforth ON. The morphology of human cervix. Clin Obstet Gynecol 1983:26(1):7-13.

[6] Olah KS. Gee H. Brown JS. Cervical contractions: the response of the cervix to oxytocin stimulation in the latent phase of labour. Br J Obstet Gynaecol 1993; 100:635-40.

[7] Wolfs GMJA. Van Leeuwen M. Electromyographic observations on the human uterus during labour. Acta Obstet Gynecol Scand Suppl 1979;90: 1-62.

[8] Devedeux D. Marque C. Mansour S. Germain G. Duchene J. Uterine 
electromyography: a critical review. Am JObstet Gynecol 1993: 168: 1636-53.

[9] Pajntar M. Roškar E. Rudel D. Electromyographic observations on the human cervix during labour. Am Obstet Gynecol 1987:156:691-7.

[10] Pajntar M. Verdenik 1. Electromyographic activity in cervices with very low Bishop score during labor. Int J Gynecol Obstet 1995;49:277-81.

[11] Pajntar M. Rudel D. Changes in electromyographic activity of the cervix after stimulation of labour with oxytocin. Gynecol Obstet Invest 1991:31:204-7.

[12] Troedsson MHT. Wistrom AOG, Liu IKM, Ing M. Pascoe J.

Thurmond M. Registration of myometrial activity using multiple site electromyography in cyclic mares. J Reprod Fertil 1993;99(2):299306.

[13] Ramondt J. Van Kooten C, Verhoeff A. Wallenburg HCS. Computer analysis of mechanical and electrical uterine activity. Med Biol Eng Comput 1986:24:3515. 